\title{
Chaotic Time-Reversed Acoustics: Sensitivity of the Loschmidt Echo to Perturbations
}

\author{
B.T. TAddese ${ }^{a, b, c}$, M.D. Johnson ${ }^{d}$, J.A. HarT ${ }^{b, e}$, T.M. Antonsen, JR. ${ }^{b, c, e}$, E. OtT $^{b, c, e}$ \\ AND S.M. ANLAGE ${ }^{a, b, c}$ \\ ${ }^{a}$ Center for Nanophysics and Advanced Materials, Physics Department, University of Maryland \\ College Park, MD 20742-4111, USA \\ ${ }^{b}$ Physics Department, University of Maryland, College Park, MD 20742-4111, USA \\ ${ }^{c}$ Department of Electrical and Computer Engineering, University of Maryland, College Park, MD 20742, USA \\ ${ }^{d}$ Department of Physics, University of California, Santa Barbara, CA 93106, USA \\ ${ }^{e}$ Institute for Research in Electronics and Applied Physics, University of Maryland \\ College Park, MD 20742-3511, USA
}

\begin{abstract}
We experimentally demonstrate a new acoustic sensor based on the concept of quantum mechanical scattering fidelity and the Loschmidt echo applied to classical acoustic waves in air. The sensor employs a one-recording-channel time-reversal mirror that exploits spatial reciprocity to sensitively measure the classical analog of the scattering fidelity of an enclosed region. The experiments are carried out in a stairwell using a simple speaker and microphone. The input is a $7.0 \mathrm{kHz}$ signal that is amplitude modulated with a $1 \mathrm{~ms}$ long pulse. We examine the sensitivity of the time-reversed reconstructed pulse to phase noise, long term drift, and to typical perturbations caused by the rotation of an object in the scattering environment.
\end{abstract}

PACS numbers: 03.55.Kk, 05.45.Mt, 43.58.+z, 43.60.+d

\section{Introduction}

Classically chaotic systems show exponential sensitivity to small changes in initial conditions. Such sensitivity makes chaotic dynamics conceptually attractive for use as a sensor of very small changes. However, many sensors and detectors operate by sending out and receiving wave excitations. For example SONAR and RADAR detect the presence of an object by sending out a wave disturbance and measuring a reflected signal, and using information from the reflected signal to determine the range and motion of the object. However, one can imagine other types of wave scattering, including those that have an underlying chaotic classical limit, which may be sensitive to objects in a different way. In particular, if one imagines a scattering system whose closed counterpart shows chaotic dynamics in the limit of small wavelength, the wave scattering properties might be extremely sensitive to small perturbations of the system. This idea motivates our development of a new sensor paradigm to exploit the sensitivity of wave scattering systems to small perturbations of the scattering environment.

However, linear wave systems cannot be chaotic. The concept of diverging trajectories makes no sense in the case of linear waves [1]. Nevertheless wave systems that have chaotic dynamics in the classical (small wavelength) limit can show unique properties. The study of wave systems whose classical dynamics is chaotic is known as "wave chaos" or "quantum chaos" [2]. In particular, these wave-chaotic systems have eigenfunctions whose statistical properties are well described as those of a random superposition of plane waves (the Berry hypothesis [3]). This property can be exploited to create an elegant and general statistical theory of the scattering properties of wave chaotic systems. In related work, we have created a random coupling model to understand the frequency-domain and time-domain properties of wave chaotic systems [4-7], and this model has been tested through experiments on a microwave resonator $[8,9]$.

Wave chaotic systems have wave scattering properties that are quite sensitive to small perturbations of the scattering environment. One measure of this sensitivity was developed in the context of quantum mechanics, where it is known as fidelity [10]. Quantum fidelity is a measure of how sensitive the dynamics of a quantum mechanical system is to small perturbations of its Hamiltonian. It can be defined as follows. A system is prepared in a given initial state $|\psi(0)\rangle$, propagated forward in time under an unperturbed time-reversible Hamiltonian $H$ to some time $t,|\psi(t)\rangle=U(t)|\psi(0)\rangle$ where $U(t)=\exp (-\mathrm{i} H t / \hbar)$ is the time evolution operator. At that time the evolution is stopped and the system is prop- 
agated backward in time under a perturbed Hamiltonian $H+H^{\prime}$ to create a new state $U^{\prime}(-t) U(t)|\psi(0)\rangle$ where $U^{\prime}(-t)=\exp \left[\mathrm{i}\left(H+H^{\prime}\right) t / \hbar\right]$. The overlap of this forward and backward propagated state with the initial state is known as the fidelity, $f_{H^{\prime}}(t)=\left\langle\psi(0)\left|U^{\prime}(-t) U(t)\right| \psi(0)\right\rangle$. The fidelity is unity in the absence of perturbations for any $H$ and $t$. However, in the presence of perturbations the fidelity will decay with $t$ at a rate depending on $H$ and the perturbation. Fidelity is also known as the Loschmidt echo (LE) [10], and thus makes connection to spin-echo experiments widely used in nuclear magnetic resonance [11].

Considerable progress to measure the LE with classical waves has been made by the development of "time-reversal mirrors" for classical waves in acoustics $[12,13]$ and electromagnetics [14-16]. Such mirrors collect and record a propagating wave as a function of time, and at some later time propagate it in the opposite direction in a time-reversed fashion. In general it is not possible to mirror all waves in this manner. However, this problem is mitigated considerably in the special case of a billiard system with classically chaotic ray dynamics. Under these conditions a single-channel time-reversal mirror can very effectively approximate the conditions required to implement the "Loschmidt echo" definition of fidelity $[17,16]$. Further simplifications can be gained by making use of spatial reciprocity of the wave equation to simplify the implementation of a "Loschmidt echo" measure of fidelity. Here we present a new sensor paradigm for classical-wave-based sensors by measuring the scattering fidelity of a ray-chaotic billiard system through the coherent time-reversed reconstruction of an excitation pulse which can detect small changes in scattering.

\section{Experiment}

A Loschmidt echo scattering fidelity experiment is demonstrated using acoustic waves in a 2-story-tall enclosed stairwell, roughly $6 \mathrm{~m}$ deep $\times 2.5 \mathrm{~m}$ wide $\times 6.5 \mathrm{~m}$ tall [18]. A desktop speaker and microphone (Samson $\mathrm{C} 01 \mathrm{U}$ ) inside the stairwell are connected to a controlling computer stationed outside the enclosure (see Fig. 1). A $7 \mathrm{kHz}$ sound wave that is amplitude modulated with a $1 \mathrm{~ms}$ long pulse is broadcast into the stairwell. The response of the stairwell is recorded at the location of the microphone. The signal recorded is quite sensitive to the geometry of scattering objects inside the stairwell, and is called a sona. Operation of a time reversal mirror would demand that we next interchange the location of the microphone and the speaker before broadcasting a time reversed sona back into the enclosure. However, spatial reciprocity of the acoustic wave equation, which states that interchanging the location of a source and a receiver does not alter what is recorded, is used to simplify the experiment. The time reversed sona broadcast retraces its ray trajectories in the stairwell in a time reversed manner, and collapses on the microphone reconstructing a time reversed version of the original pulse.

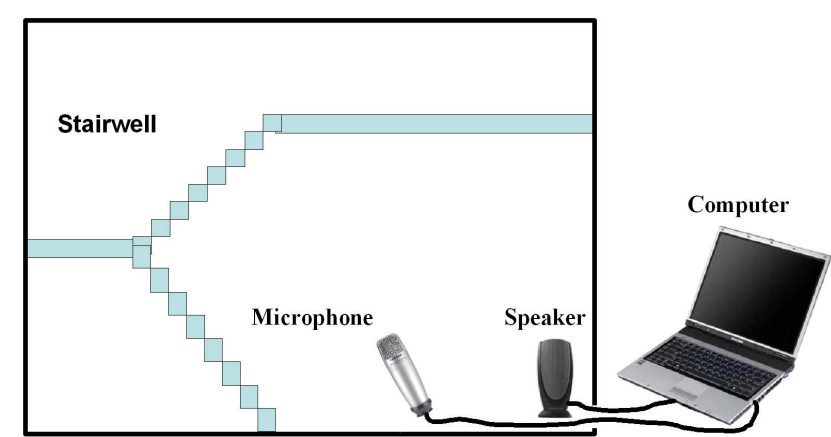

Fig. 1. Schematic diagram of the acoustic time reversal mirror experiment, which utilizes spatial reciprocity for sound waves in a stairwell. A computer outside the reverberant cavity is connected to a speaker and a microphone that are placed inside the enclosure many wavelengths apart.

The above mentioned experimental procedure to reconstruct a time reversed pulse is used to investigate the following issues. The robustness of the acoustic time reversal mirror is investigated by broadcasting a time reversed sona after numerically modifying its phase information. The long term drift induced by uncontrollable environmental changes to the cavity is studied by reconstructing time reversed pulses successively over a long time period using a single sona signal recorded at an earlier time. The feasibility of the acoustic time reversal mirror as a sensor is then demonstrated as follows. A time reversed pulse is reconstructed in a stairwell with a given configuration of objects, then the same sona signal is used to reconstruct a time reversed pulse after we rotate a rectangular box to a new orientation in the stairwell. The time reversed pulses reconstructed before and after the perturbation to the cavity are compared with a goal of detecting the perturbation.

\section{Results}

\subsection{Phase noise added to the sona}

The robustness of the time reversal mirror to phase noise/manipulation that corrupts the sona signal is studied. We performed an experiment in which the digitized sona signal is numerically modified before it is time reversed and broadcasted back into the cavity it was collected from. Specifically, the sona is Fourier transformed to get its magnitude and phase information in the frequency domain (with a frequency step of $2 \mathrm{~Hz}$ between data points). Gaussian distributed random numbers with zero mean and a given standard deviation, which is systematically varied from 0 to $\pi$, are added to the phase of the Fourier transform of the sona. The unaltered magnitude information, and the modified phase information are used to generate a modified sona signal using inverse Fourier transform. This modified sona is then time reversed and broadcasted into the cavity, and the peak to peak amplitude of the reconstructed pulse is measured. 
The peak to peak amplitude of the reconstructed pulse is observed to diminish as the standard deviation of the phase noise is increased (see Fig. 2). This result is also seen using a theoretical model that applies similar phase noise to the sona signal.

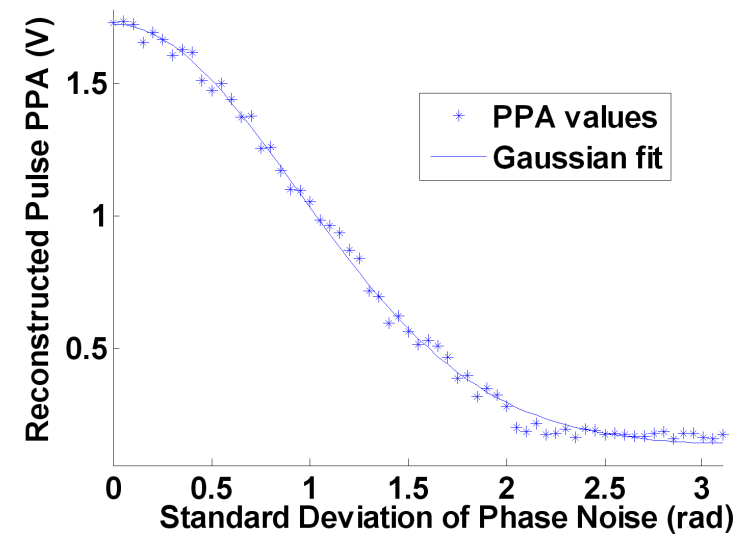

Fig. 2. A plot of peak-to-peak amplitude (PPA) of the reconstructed pulse amplitude in volts versus the standard deviation of the Gaussian phase noise distribution in radians. Gaussian distributed random numbers with zero mean and a standard deviation, which is systematically varied between 0 and $\pi$, are added to the phase of the Fourier transform of the sona signal. This effectively scales the reconstructed pulse by a Gaussian function of the standard deviation of the underlying phase noise.

Because the system is linear, the effect of phase noise on the sona can be directly calculated. The sona can be expressed as a Fourier transform as $S(t)=$ $\int \mathrm{d} \omega S(\omega) \mathrm{e}^{\mathrm{i} \omega t}$. With added phase noise, distributed as

$$
P_{\Delta \theta}(\theta)=\frac{1}{\sqrt{2 \pi(\Delta \theta)^{2}}} \mathrm{e}^{-\frac{\theta^{2}}{2(\Delta \theta)^{2}}},
$$

the modified sona becomes

$$
S_{\text {noisy }}(t)=\int \mathrm{d} \omega \tilde{S}(\omega) \int \mathrm{d} \theta \mathrm{e}^{\mathrm{i} \omega t} \mathrm{e}^{\mathrm{i} \theta} P(\theta) .
$$

Carrying out the integral over the random variable leads to

$$
S_{\text {noisy }}(t)=\int \mathrm{d} \omega \tilde{S}(\omega) \mathrm{e}^{\mathrm{i} \omega t} \mathrm{e}^{-\frac{(\Delta \theta)^{2}}{2}} .
$$

Clearly the sona signal is reduced in magnitude by an amount that depends on the width of the Gaussian distribution $\Delta \theta$.

The degradation of the sona signal translates into degradation of the reconstructed pulse, as shown in Fig. 2. The peak-to-peak amplitude of the reconstructed pulse versus width of the Gaussian phase noise distribution is fit to a Gaussian function as

$$
V_{\mathrm{p}-\mathrm{p}}(\Delta \theta)=V_{0}+A \mathrm{e}^{-B \frac{(\Delta \theta)^{2}}{2}},
$$

and the three parameters $V_{0}, A$, and $B$ are allowed to vary. The result shows an excellent fit with $V_{0}=0.14 \mathrm{~V}$, $A=1.58 \mathrm{~V}$, and $B=1.14$. The value of $B$ is very close to the expected value of 1 , while the offset value $V_{0}$ is the background level in the extremely noisy limit.

\subsection{Long-term drift of the reconstructed pulse}

As the time reversed sona signal is periodically broadcast into the cavity, the reconstructed time reversed pulse consistently reproduces itself up to some statistical measurement fluctuation. Particularly, we look at the fluctuations in the peak-to-peak amplitude of the reconstructed pulse as a single time reversed sona is periodically broadcasted into a nominally unperturbed cavity every $10 \mathrm{~s}$ over a time period of $20 \mathrm{~min}$ (see Fig. 3). For short time

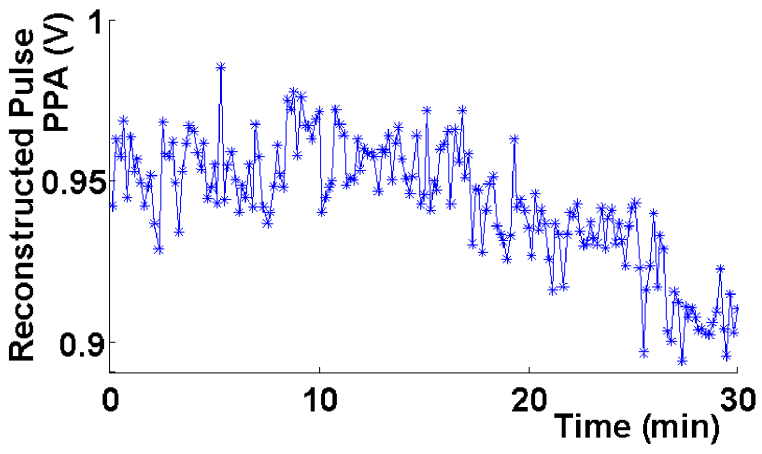

Fig. 3. Long term drift in the PPA in volts of the reconstructed time reversed pulse in the stairwell. The PPA exhibits a drift as the reverberant cavity and air medium go through thermally-induced changes in time.

periods, the statistical variation of the peak-to-peak amplitude is about $\pm 2 \%$. However, there are uncontrollable changes to the cavity over long time periods, such as thermal expansion or perhaps changes in temperature of the air, and these play a role in the drift of the reconstructed time reversed pulse amplitude on longer time scales (>10 $\mathrm{min})$.

\subsection{Effect of a volume-preserving perturbation}

Finally, we test the feasibility of the acoustic time reversal mirror to detect volume preserving perturbations to the cavity by comparing reconstructed pulses before and after a specific perturbation of the scattering environment. The perturbation we perform involves rotating a rectangular cardboard box (with a volume of $30 \times 60 \times 80 \mathrm{~cm}^{3}$ ), located inside the stairwell, by 90 degrees. The reconstructed time-reversed pulses before and after perturbation are compared in one of the following ways: (1) the peak-to-peak amplitude of the two pulses are compared, or (2) the normalized cross correlation between the two reconstructed pulses is calculated. The peak-to-peak amplitude of the reconstructed pulse drops from $1.11 \mathrm{~V}$ to $0.92 \mathrm{~V}$ upon the perturbation; this is a reduction by $17 \%$ (see Fig. 4), which is significant compared to the typical statistical fluctuation of about $\pm 2 \%$ between nominally identical reconstructed pulses (Fig. 3). On the other hand, the normalized cross correlation of the pulses before and after perturbation is 


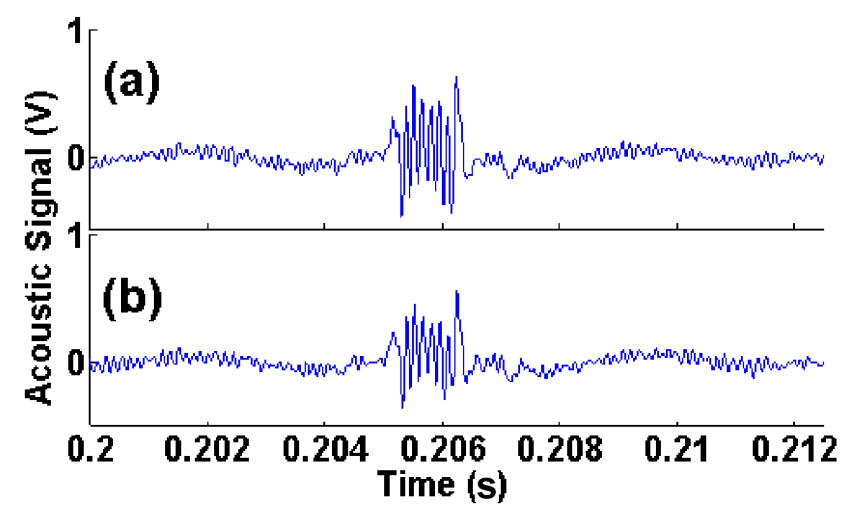

Fig. 4. The effect of a volume preserving perturbation on the reconstructed time reversed (TR) pulse. The TR pulses $(\mathrm{a})$ before $(\mathrm{PPA}=1.11 \mathrm{~V})$ and $(\mathrm{b})$ after $(\mathrm{PPA}=$ $0.92 \mathrm{~V})$ perturbation have a normalized correlation of $93 \%$, but the PPA drops by $17 \%$. The perturbation is done by rotating a rectangular box $\left(30 \times 60 \times 80 \mathrm{~cm}^{3}\right)$ by ninety degrees inside the reverberant cavity (stairwell).

93\%. From this and many other measurements [18] it is clear that monitoring the decrease in the peak-to-peak amplitude of the reconstructed time-reversed pulse is not only computationally simpler but it is also a statistically more reliable mechanism to detect perturbations.

\section{Discussion}

The advantage of using time reversal mirrors for sensing applications stems from the underlying chaos present in the closed ray-dynamic version of the scattering system. The chaos implies that a broadcast pulse reflects off of most parts of the cavity before being recorded by the microphone. The time reversed sona also explores the cavity in a time reversed manner before reconstructing as a time reversed pulse at the microphone. Any changes in the boundary conditions of the cavity break time reversal invariance, resulting in a degradation of the reconstruction of the pulse. We have shown that this degradation manifests itself clearly as a drop in the peak to peak amplitude of the reconstructed time reversed pulse.

Because of the chaotic nature of the ray dynamics of the enclosure, the waves broadcast are "space filling" and reach all parts of the cavity. This has the advantage of increasing the sensitivity to scattering perturbations throughout the volume. The disadvantage is that the sensor cannot easily identify the location and size of the perturbation. Further work is needed to refine the sensor to reveal more detailed information about the location and nature of the perturbation giving rise to the signal [18].

\section{Conclusions}

We have developed a new class of sensors that exploits the scattering properties of wave chaotic systems along with time-reversal invariance and spatial reciprocity of the wave equation. This sensor measures the classical analog of the quantum fidelity to detect small changes in a scattering environment.

\section{Acknowledgments}

We thank Jen-Hao Yeh for technical assistance and advice. This work is supported by an ONR MURI entitled "Exploiting Nonlinear Dynamics for Novel Sensor Networks", grant N000140710734, an ONR DURIP grant N000140710708, and AFOSR under grant FA95500710049.

\section{References}

[1] R.K. Snieder, J.A. Scales, Phys. Rev. E 58, 5668 (1998).

[2] H.-J. Stockmann, Quantum Chaos, Cambridge University Press, New York 1999.

[3] M.V. Berry, in: Chaotic Behavior of Deterministic Systems, Eds. G. Iooss, R.H.G. Helleman, R. Stora, North-Holland, Amsterdam 1991, p. 171.

[4] X. Zheng, T.M. Antonsen Jr., E. Ott, Electromagnetics 26, 3 (2006).

[5] X. Zheng, T.M. Antonsen Jr., E. Ott, Electromagnetics 26, 37 (2006).

[6] J. Hart, T.M. Antonsen, Jr., E. Ott, Phys. Rev. E 79, 016208 (2009).

[7] J.A. Hart, T.M. Antonsen, E. Ott, Phys. Rev. E 80, 041109 (2009).

[8] S. Hemmady, X. Zheng, E. Ott, T.M. Antonsen, S.M. Anlage, Phys. Rev. Lett. 94, 014102 (2005).

[9] S. Hemmady, X. Zheng, T.M. Antonsen, E. Ott, S.M. Anlage, Phys. Rev. E 71, 056215 (2005).

[10] T. Gorin, T. Prosen, T.H. Seligman, M. Žnidarič, Phys. Rep. 435, 33 (2006).

[11] C.P. Slichter, Principles of Magnetic Resonance, 3rd ed., Springer-Verlag, New York 1990, p. 46.

[12] M. Fink, Contemp. Phys. 37, 95 (1996).

[13] M. Fink, D. Cassereau, A. Derode, C. Prada, P. Roux, M. Tanter, J.-L. Thomas, F. Wu, Rep. Prog. Phys. 63, 1933 (2000).

[14] G. Lerosey, J. de Rosny, A. Tourin, A. Derode, G. Montaldo, M. Fink, Phys. Rev. Lett. 92, 193904 (2004).

[15] G. Lerosey, J. de Rosny, A. Tourin, A. Derode, M. Fink, Appl. Phys. Lett. 88, 154101 (2006).

[16] S.M. Anlage, J. Rodgers, S. Hemmady, J. Hart, T.M. Antonsen, E. Ott, Acta Phys. Pol. A 112, 569 (2007).

[17] C. Draeger, M. Fink, Phys. Rev. Lett. 79, 407 (1997).

[18] B.T. Taddese, J. Hart, T.M. Antonsen, E. Ott, S.M. Anlage, submitted to Appl. Phys. Lett. 95, 114103 (2009). 